\title{
Double-PulSE Two-Micron IPDA LiDAR Simulation FOR Airborne CARbon Dioxide Measurements
}

\author{
Tamer F. Refaat*, Upendra N. Singh, Jirong Yu, and Mulugeta Petros \\ NASA Langley Research Center, Hampton, VA 23681, USA,
}

*Email: tamer.f.refaat@nasa.gov

\begin{abstract}
An advanced double-pulse 2- $\mu \mathrm{m}$ integrated path differential absorption lidar has been developed at NASA Langley Research Center for measuring atmospheric carbon dioxide. The instrument utilizes a state-of-the-art 2- $\mu \mathrm{m}$ laser transmitter with tunable on-line wavelength and advanced receiver. Instrument modeling and airborne simulations are presented in this paper. Focusing on random errors, results demonstrate instrument capabilities of performing precise carbon dioxide differential optical depth measurement with less than 3\% random error for single-shot operation up to $11 \mathrm{~km}$ altitude. This study is useful for defining $\mathrm{CO}_{2}$ measurement weighting function for adaptive targeting, instrument setting, validation and sensitivity trade-offs.
\end{abstract}

\section{INTRODUCTION}

Carbon dioxide $\left(\mathrm{CO}_{2}\right)$ is an important greenhouse gas, which critically affects Earth's climate. NASA has being developing several passive and active remote sensing instruments targeting precise monitoring of atmospheric $\mathrm{CO}_{2}$ [1-2]. Recent efforts at NASA Langley Research Center (LaRC) focused on developing, integrating and validating a unique double-pulse $2-\mu \mathrm{m}$ integrated path differential absorption (IPDA) lidar for $\mathrm{CO}_{2}$ remote sensing [3-4]. This instrument utilizes a state-of-the-art, compact, hardened and highly efficient laser transmitter. The laser transmitter is based on solid state Ho:Tm:YLF crystal technology. The transmitter produces two Qswitched laser pulses at $2-\mu \mathrm{m}$ wavelength for every $792 \mathrm{~nm}$ pump pulse. Targeting the $\mathrm{CO}_{2} \mathrm{R} 30$ line, the first $2-\mu \mathrm{m}$ pulse is set to the on-line wavelength. The on-line wavelength can be tuned and locked to any position from $1 \mathrm{GHz}$ to $6 \mathrm{GHz}$ with $1 \mathrm{GHz}$ step relative to the $\mathrm{CO}_{2} \mathrm{R} 30$ line peak toward longer wavelength. The transmitted online pulse energy is set to $90 \mathrm{~mJ}$ with $200 \mathrm{nsec}$ pulse width. The second 2- $\mu \mathrm{m}$ pulse, produced within $200 \mu \mathrm{sec}$ from the first, is set to a fixed offline wavelength with $45 \mathrm{~mJ}$ pulse energy and 350 nsec pulse width. The laser repetition rate is set to $10 \mathrm{~Hz}$. The IPDA receiver consists of a high quality $0.4 \mathrm{~m}$ diameter Newtonian telescope, which focuses the radiation, reflected from a hard target, onto $300-\mu \mathrm{m}$ diameter detectors through field-imaging aft-optics. The detectors of choice are the extended range InGaAs pin photodiodes (Hamamatsu; G5853-23), the outputs of which are applied to trans-impedance amplifiers (TIA) (Femto; DHPCA-100). Similar detector and TIA combination is integrated inside the transmitter enclosure for laser pulse energy monitoring [5]. Signals from the hard target and the laser energy monitor are digitized and recorded using 12-Bit, $200 \mathrm{MS} / \mathrm{s}$ (Agilent; U1066A) and 10-Bit, 500 MS/s (Agilent; U1065A) digitizers, respectively.

$\mathrm{CO}_{2}$ double-path differential optical depth, $d O D$, is the main IPDA product. Modeling the doublepulse 2- $\mu \mathrm{m}$ IPDA lidar instrument is the focus of this paper. IPDA lidar modeling is critical for instrument validation and simulation. Instrument validation is conducted by comparing and evaluating the IPDA measurement versus model calculations. Besides, modeling provides the necessary tools for converting $d O D$ into $\mathrm{CO}_{2}$ weighted average column dry-air volume-mixing ratio that can be compared to the gas in-situ sensors. Instrument simulations are necessary to define the measurement sensitivity and limits against the various IPDA parameters settings, such as on-line wavelength and gain selections, and operating conditions, such as airborne platform altitude, ground conditions and environment. In the presented model, six different altitudes are assumed, spanning from $1 \mathrm{~km}$ to 11 $\mathrm{km}$, with $2 \mathrm{~km}$ step. This allows fast scaling to any altitude assuming a small aircraft platform, such as the NASA B-200. This IPDA model is based on the US standard atmosphere model which provides typical meteorological profiles 
[1]. End products include operation charts, which produce estimates of return signal strength, $d O D$, signal-to-noise ratio (SNR) and random error.

\section{SPECTROSCOPY}

Figure 1 shows the composite absorption spectra of $\mathrm{CO}_{2}$ around the $\mathrm{R} 30$ line, located at 2050.9670 $\mathrm{nm}$. The figure also compares similar spectra for water vapor $\left(\mathrm{H}_{2} \mathrm{O}\right)$, which is the dominant interfering gas for $\mathrm{CO}_{2}$ measurement at $2-\mu \mathrm{m}$. The spectra were derived using HITRAN for line parameters, assuming Voigt line profile at two altitudes [1]. In this calculation, 5550 and 1816 lines were included for $\mathrm{CO}_{2}$ and $\mathrm{H}_{2} \mathrm{O}$, respectively. The vertical lines mark the different IPDA on-line tuning and off-line positions. Due to selected wavelength positions, $\mathrm{H}_{2} \mathrm{O}$ differential cross section is negligible with respect to $\mathrm{CO}_{2}$, which results in minimal molecular interference error. Focusing on the selected operational wavelengths, the variation of the absorption cross section, $\sigma$, versus altitude is shown in figure 2 .

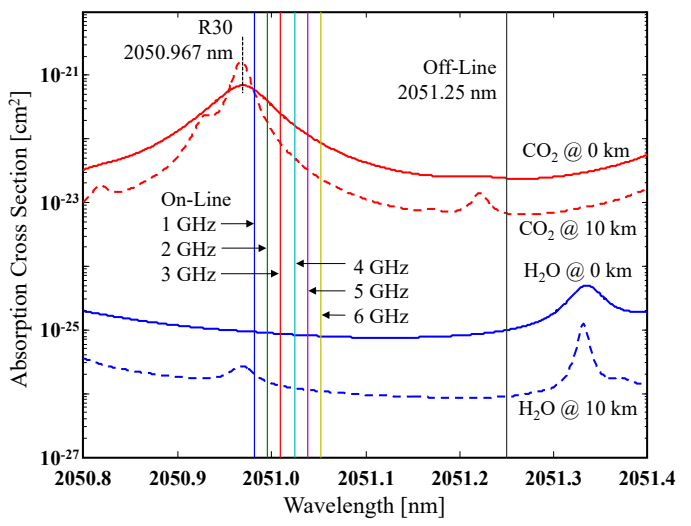

Figure 1. $\mathrm{CO}_{2}$ and $\mathrm{H}_{2} \mathrm{O}$ absorption spectra and IPDA operational wavelength settings.

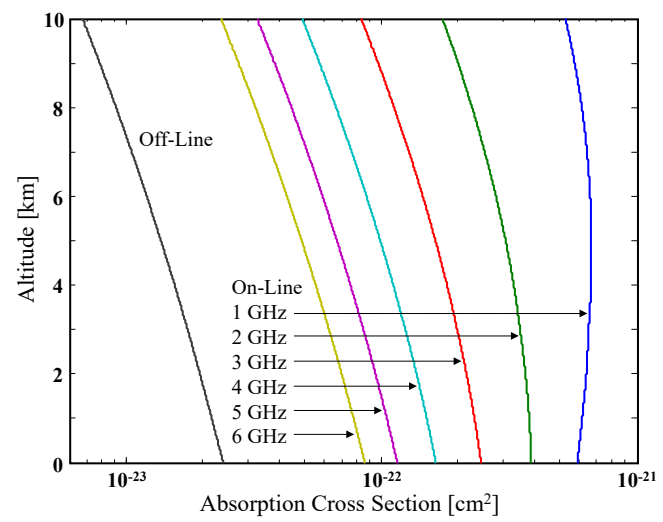

Figure 2. $\mathrm{CO}_{2}$ absorption cross section versus altitude.

\section{IPDA LIDAR SIMULATION}

IPDA lidar operation relies on the hard target lidar equation, where the return power, $P$ (in Watt) is given by the equation

$$
P=\eta_{r} \cdot \varphi_{r} \cdot \frac{A}{\left(R_{A}-R_{G}\right)^{2}} \cdot \frac{E}{t} \cdot \frac{\rho}{\pi} \cdot T
$$

for a nadir airborne instrument at a fixed altitude, $R_{A}$, and ground elevation, $R_{G}$, above sea level. In this equation, $\eta_{r}$ is the receiver optical efficiency, $\varphi_{r}$ is the overlap function, $A$ is the telescope area, $E$ is the transmitted energy, $t$ is the transmitted pulse width, $\rho$ is the surface reflectivity and $T$ is the total transmission, defined at the operating wavelength, $\lambda$. For a given wavelength, the total transmission was calculated according to

$$
T=\exp \left(-O D_{c d}-O D_{w v}-O D_{A}\right)
$$

where $O D_{c d}, O D_{w v}$ and $O D_{A}$ are the $\mathrm{CO}_{2}, \mathrm{H}_{2} \mathrm{O}$ and aerosol optical depths, respectively. Aerosol optical depth was obtained through standard model [1]. Appling US standard atmosphere model, the $\mathrm{CO}_{2}$ double-path optical depth is calculated from

$$
O D_{c d}=2 \int_{R_{G}}^{R_{4}} \sigma_{c d} \cdot N_{c d} \cdot d r
$$

where $N_{c d}$ is the $\mathrm{CO}_{2}$ number density. $N_{c d}$ vertical profile was updated from the US standard model to a recent surface dry mixing ratio of $397 \mathrm{ppm}$. $\mathrm{H}_{2} \mathrm{O}$ double-path optical depth was calculated using the same equation. Figure 3 shows the total transmission profiles obtained for the operating wavelengths from different airborne altitudes.

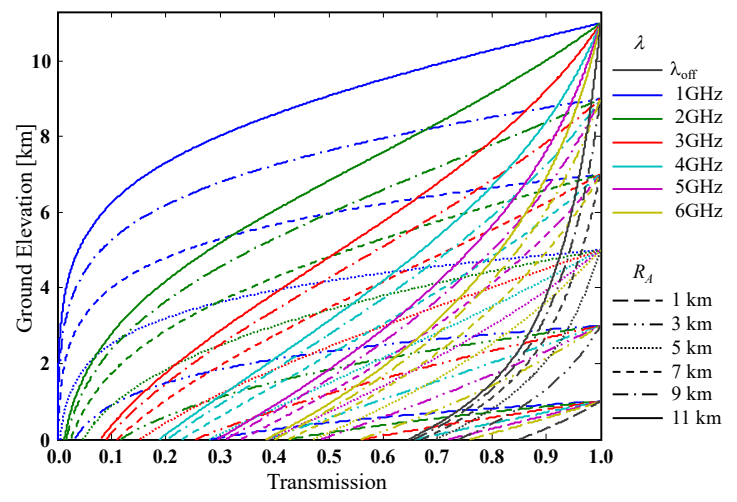

Figure 3. IPDA lidar nadir transmission at different operating on-line settings and altitudes. 


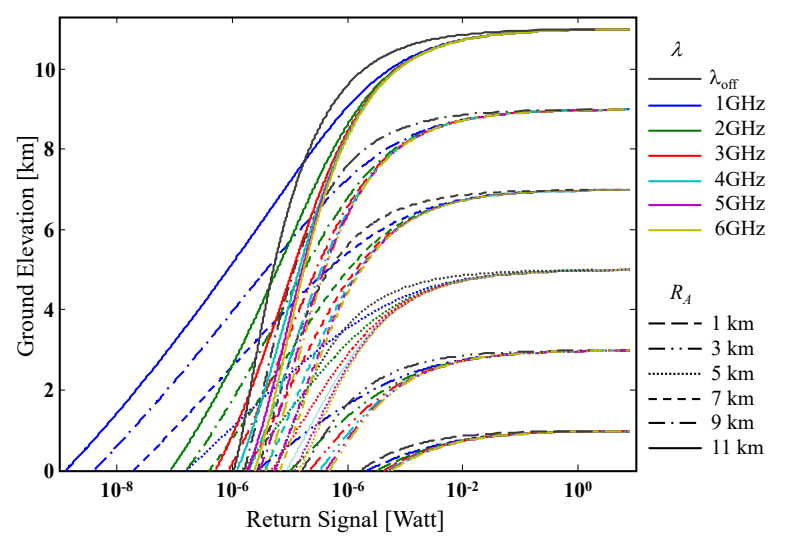

Figure 4. IPDA lidar nadir return power from ocean surface at different operating conditions.

The presented transmissions were substituted in equation (1) to estimate the hard target return power, as shown in figure 4 . For this calculation $65 \%$ receiver optical efficiency, with full overlap $\left(\varphi_{r}=1\right)$, and moderate ocean reflectivity were considered (i.e., $R_{G}=0$ and $\rho=0.08$ ). With detector responsivity set to $1.15 \mathrm{~A} / \mathrm{W}$, the return power chart is useful to set proper TIA gains that match the output voltage to the digitizer input limits. Besides, it gives a quick estimate for critical IPDA performance parameters, such as SNR and random error.

\section{IPDA LIDAR PERFORMANCE}

Figure 5 shows the 2- $\mu \mathrm{m}$ IPDA lidar, single-shot power SNR variation with ground elevation for different airborne altitude and wavelength settings. SNR were calculated as the ratio of the return power, shown in figure 4 , to the total noise power. The total noise power was obtained by combining the instrument fixed noise and signal dependent shot noise. Fixed noises include electronic noises and background radiation. Dominant electronic noises sources, such as detector dark current and TIA feedback Johnson noise, input current and voltage noises and coupling noise, were considered. Other electronic noise sources, such as digitizer noise and interference, were found weak and neglected. The presented SNR is based on the IPDA lidar receiver assuming ideal performance. SNR could be deteriorated due other factors such as speckles and pulse energy fluctuations of the transmitted laser. Both of these effects were minimized in the system design and thus neglected in this analysis.

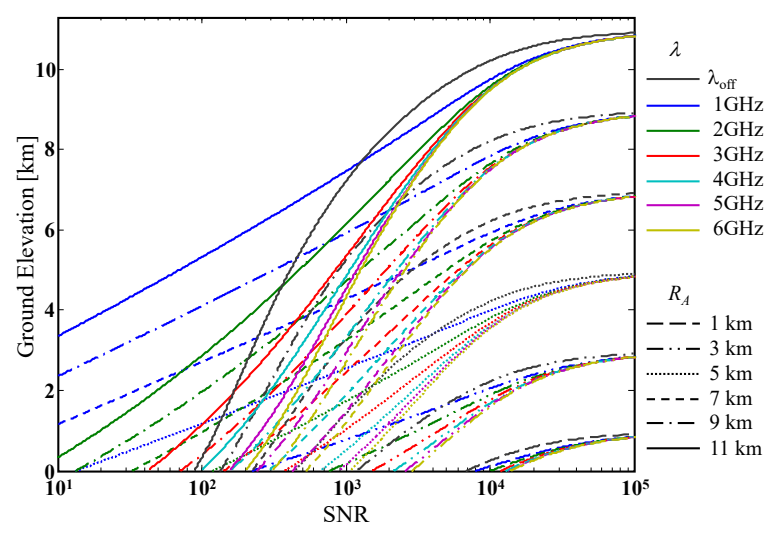

Figure 5. IPDA lidar SNR estimates at different operating conditions.

For example, speckle SNR could be enhanced by the transmitted laser beam divergence and the sampling time. Besides, laser pulse fluctuation effects could be minimized by proper energy monitoring and normalization per shot [5].

During the 2- $\mu \mathrm{m}$ IPDA operation the selected online and off-line return pulses are collected per shot. During data analysis, both on- and off-lines pulses are reduced to obtain the $\mathrm{CO}_{2} d O D$ measurement, which according to equation 3 , can be modeled as

$d O D_{c d}=O D_{c d}\left(\lambda_{o n}\right)-O D_{c d}\left(\lambda_{o f f}\right)$

where $\lambda_{o n}$ and $\lambda_{\text {off }}$ are the on-line and off-line wavelengths, respectively. The uncertainty associated with $d O D$ measurement, $\delta(d O D)$, can be estimated from the SNR of the return signals using the equation [1]

$\delta\left(d O D_{c d}\right)=\sqrt{\frac{S N R_{o n}^{-2}+S N R_{o f f}^{-2}}{N}}$

This $d O D$ uncertainty represents the measurement random error caused by the receiver. In the above equation $S N R_{\text {on }}$ and $S N R_{\text {off }}$ are the on- and off-line return signal SNR, respectively, presented in figure 5, and $N$ is the number of shot averages. Figure 6 shows the variation of the $\mathrm{CO}_{2}$ doublepath differential optical depth with ground elevation for different flight altitude and operating on-line settings. The corresponding random errors are shown in figure 7, for single-shot operation.

The random error was calculated as the ratio of the measurement uncertainty, from equation (5) to differential optical depth, from equation (4). 
Results indicate the possibility of measuring $\mathrm{CO}_{2}$ optical depth accurately with less than $3 \%$ singleshot random error (i.e., without averaging). From equation (5), shot averaging would reduce the error further. For example, operating the IPDA instrument from a platform at $1 \mathrm{~km}$ altitude, a minimum error of $0.02 \%$ can be achieved with 1 $\mathrm{GHz}$ on-line setting. At this setting, both on- and off-line return powers would be within $100 \mu \mathrm{W}$ with $d O D$ around unity and $10^{4} \mathrm{SNR}$. Operating the instrument from a platform at $11 \mathrm{~km}$ altitude, $4 \mathrm{GHz}$ would result in a minimum error of $1.2 \%$ at sea level that is dominated by the off-line SNR. This error would reduce to $0.12 \%$ with $10 \mathrm{sec}$ shot average. For the same altitude, $3 \mathrm{GHz}$ would result in lower errors than $4 \mathrm{GHz}$ for ground elevation above $800 \mathrm{~m}$.

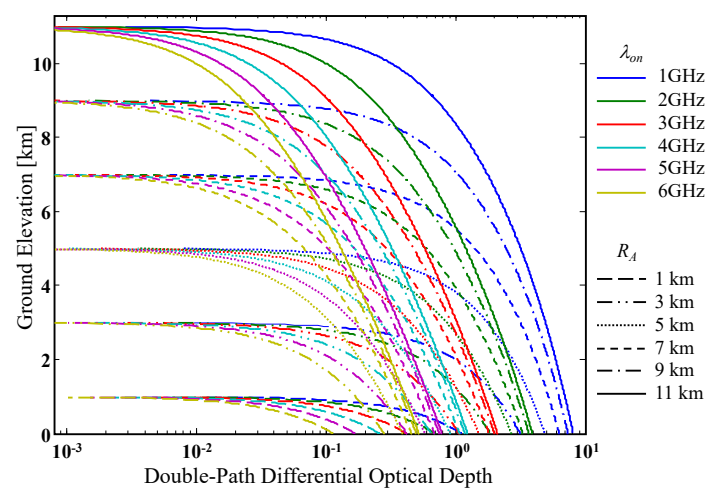

Figure 6. Double-pulse 2- $\mu \mathrm{m} \mathrm{CO}_{2}$ IPDA lidar doublepath differential optical depth.

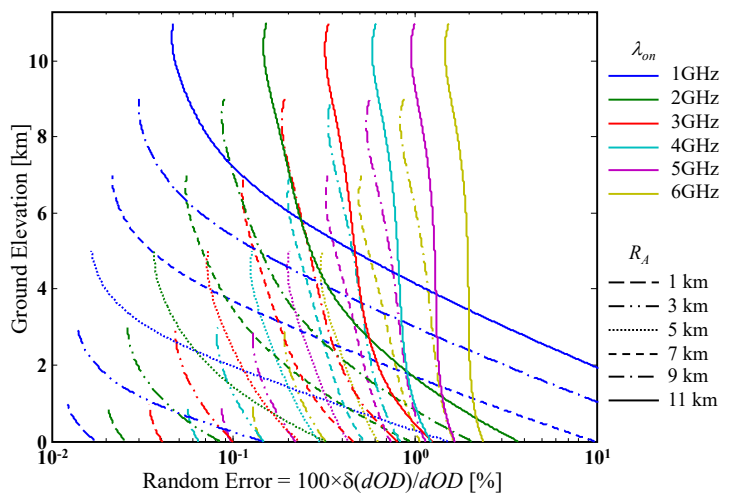

Figure 7. $\mathrm{CO}_{2}$ differential optical depth random error, calculated assuming single-shot operation.

\section{CONCLUSIONS}

An advanced double-pulse 2- $\mu$ m IPDA lidar has been developed at NASA LaRC for measuring atmospheric $\mathrm{CO}_{2}$. The instrument utilizes a stateof-the-art $2-\mu \mathrm{m}$ tunable laser transmitter and advanced receiver. IPDA lidar modeling is useful for defining the instrument setting and measurement validation and sensitivity trade-offs. Airborne simulations resulted in less than 3\% random error for single-shot operation up to 11 $\mathrm{km}$ altitude. This demonstrates the 2- $\mu \mathrm{m}$ IPDA lidar capability of performing precise $\mathrm{CO}_{2}$ measurement from airborne platforms. This model has been used to validate the $\mathrm{CO}_{2} 2-\mu \mathrm{m}$ IPDA lidar instrument performance from ground and airborne platforms as will be the presented in future studies.

\section{ACKNOWLEDGEMENT}

This work (ESTO ATI-QRS-2012, Principal Investigator: Upendra Singh, LaRC) was funded and supported by NASA Earth Science Technology Office (Program Director: George Komar and Program Manager: Parminder Ghuman).

\section{REFERENCES}

[1] T. Refaat, U. Singh, J. Yu, M. Petros, S. Ismail, M. Kavaya, K. Davis, 2015: Evaluation of an airborne triple-pulsed $2 \mu \mathrm{m}$ IPDA lidar for simultaneous and independent atmospheric water vapor and carbon dioxide measurements, Appl. Opt. 54, 1387-1398.

[2] U. Singh, B. Walsh, J. Yu, M. Petros, M. Kavaya, T. Refaat, N. Barnes, 2015: Twenty years of Tm:Ho:YLF and LuLiF laser development for global wind and carbon dioxide active remote sensing, Opt. Mater. Exp. 5, 827-837.

[3] J. Yu, M. Petros, K. Reithmaier, Y. Bai, B. Trieu, T. Refaat, M. Kavaya, U. Singh, S. Ismail, 2012: A 2-micron pulsed integrated path differential absorption lidar development for atmospheric $\mathrm{CO}_{2}$ concentration measurements," in 26th ILRC, Porto Heli, Greece.

[4]. U. Singh, J. Yu, M. Petros, T. Refaat, R. Remus, J. Fay, K. Reithmaier, 2014: Airborne 2micron double-pulsed integrated path differential absorption lidar for column $\mathrm{CO}_{2}$ measurement", Proc. SPIE 9246, 924602.

[5] T. Refaat, M. Petros, R. Remus, J. Yu, U. Singh, 2014: Laser energy monitor for doublepulsed 2- $\mu \mathrm{m}$ IPDA lidar application, Proc. SPIE 9246, 924606. 